\title{
A Modified Invasive Weed Optimization Algorithm for Time-Modulated Linear Antenna Array Synthesis
}

\author{
Aniruddha Basak, Siddharth Pal, Swagatam Das, Ajith Abraham and Vaclav Snasel
}

\begin{abstract}
Time modulated antenna arrays attracted the attention of researchers for the synthesis of low/ultra-low side lobes in recent past. In this article we propose an improved variant of a recently developed ecologically inspired metaheuristic, wellknown as Invasive Weed Optimization (IWO), to solve the real parameter optimization problem related to the design of timemodulated linear antenna arrays with ultra low Side Lobe Level (SLL), Side Band Level (SBL) and Main Lobe Beam Width (BWFN). We improvise the classical IWO by introducing two parallel populations and a more explorative routine of changing the mutation step-size with iterations. Experimental results indicate that the proposed algorithm achieves better performance over the design problem as compared to the conventional Taylor Series based method and the only known metaheuristic approach based on the Differential Evolution (DE) algorithm.
\end{abstract}

Keywords: Antenna array, invasive weed optimization, time modulation, side lobe suppression, differential evolution, particle swarm optimization, electromagnetic optimization.

\section{INTRODUCTION}

Although first proposed long back in 1960's time modulated antenna arrays have recently been revived by the researchers due to their efficiency in realizing ultra-low sidelobe levels in the far-field pattern $[1-5]$. This feature is mainly attributed to the fact that time modulated antenna arrays incorporate an additional degree of freedom in their design-the time. For an 8element slotted linear array the time modulation method and the realization of a nearly ultra-low SLL $(\sim 39.8 \mathrm{~dB})$ were first achieved by Kummer et al [2]. The general principles for analysis of time modulated antenna system were first put forward by Bickmore in [5]. Although antenna arrays of this kind have greater flexibility for design and offers significant reduction in the dynamic-range ratio of the excitation for ultralow SLLs as compared to that required in ordinary SLLs, the design of time modulated arrays is still complicated due to the presence of a multitude of sideband signals. Since these sideband signals are usually spaced at multiples of the modulation frequency, a significant portion of the radiated or received power is shifted to the sidebands.

Aniruddha Basak is with Department. of Electronics and Telecommunication Engineering, Jadavpur University , India (aniruddha_ju_etce@yahoo.com).

Siddharth Pal is with Department. of Electronics and Telecommunication Engineering, Jadavpur University, India (sidd pal2002@yahoo.com).

Swagatam Das is with Department. of Electronics and Telecommunication Engineering, Jadavpur University, India (swagatamdas19@yahoo.co.in).

Ajith Abraham is with Machine Intelligence Resarch Labs (MIR Labs), USA and Faculty of Electrical Engineering and Computer Science, VSB Technical University of Ostrava, Czech Republic (ajith.abraham@ieee.org)

Vaclav Snasel is with the Faculty of Electrical Engineering and Computer Science, VSB Technical University of Ostrava, Czech Republic ('vaclav.snasel@vsb.cz)
Certain applications demand complete removal of sideband signals and hence they should be suppressed as far as possible to improve the efficiency of array design. In [5] Yang et al. proposed a Differential Evolution (DE) based approach for the design of time modulated linear arrays with effective suppression of sideband radiation patterns. Such designs offer severe challenges to the antenna researchers and as indicated by [6] metaheuristic algorithms can be the best ways to handle them. However, to the best of our knowledge, other than DE, no other population-based metaheuristic has been applied to this kind of problems till date.

Classical derivative-based optimization techniques need a well defined starting point which should be significantly close to the final solution or they are very likely to get trapped in a local optimum. The computational drawbacks of existing numerical methods have forced the researchers all over the world to rely on metaheuristic algorithms founded on the basis of simulation of some natural phenomena. In recent past the computation cost having been reduced dramatically, researchers from diverse domains of science and engineering are drawn towards bio-mimicry and bio-inspiration, for solving computational problems and constructing intelligent systems like autonomous robots. Following the same trends, in 2006 Mehrabian and Lucas proposed the Invasive Weed Optimization (IWO) [7], a derivative free metaheuristic algorithm mimicking the ecological behaviour of colonizing weeds. Since its advent IWO has found several successful engineering applications like tuning of Robot Controller [7], Optimal Positioning of Piezoelectric actuators [8] , development of recommender system [9] , antenna configuration optimization [10], design of E-shaped MIMO antenna [11], design of compact U-array MIMO antenna [12] , DNA computing [13], and so on.

In this work we modify the classical IWO with a view to improving its final accuracy and convergence speed over multimodal fitness landscapes. We then apply the modified IWO variant to the design of linear time modulated arrays. Our algorithm attempts to minimize the SLLs at centre frequency and sideband levels simultaneously and it uses the static excitation amplitude distribution and the switch on time intervals as the parameters to optimize. Comparisons with the classical DE based approach [4], a state-of-the-art DE variant [14] and state-of-the-art version of another very popular swarm intelligence algorithm called Particle Swarm Optimization (PSO) [15] over three difficult instantiations of the design problem involving 16, 32, and 64 elements arrays reflect the superiority of the proposed approach in terms of final accuracy, computational time, and robustness. Rest of the paper is organized as follows: 
Section 2 provides an outline of the classical IWO algorithm and also describes the proposed modifications of the algorithm. Section 3 provides the formulation of the design problem in context to time modulated antenna arrays. Section 4 presents and discusses the experimental results. Finally the paper in concluded in Section 5 along with a discussion on the future avenues of research.

\section{Classical IWO AND ITS MODIFICATIONS}

Invasive Weed Optimization (IWO) is a meta-heuristic algorithm that mimics the colonizing behavior of weeds. The algorithm for IWO may be summarized as follows:

\section{A. Initialization}

A finite number of weeds are initialized at the same element position of the conventional array which has a uniform spacing of $\lambda / 2$ between neighbouring elements.

\section{B. Reproduction}

Each member of the population is allowed to produce seeds depending on its own, as well as the colony's lowest and highest fitness, such that, the number of seeds produced by a weed increases linearly from lowest possible seed for a weed with worst fitness to the maximum number of seeds for a plant with best fitness.

\section{Spatial distribution}

The generated seeds are being randomly distributed over the $d$-dimensional search space by normally distributed random numbers with mean equal to zero; but varying variance. This step ensures that the produced seeds will be generated around the parent weed, leading to a local search around each plant. However, the standard deviation (SD) of the random function is made to decrease over the iterations.

If $s d_{-}$max and $s d_{-}$min be the maximum and minimum standard deviation and if pow be a real no., then the standard deviation for a particular iteration may be given as in equation (1):

$$
s d_{\text {ITER }}=\left(\frac{\text { iter }_{\max }-\text { iter }}{\text { iter }}\right)_{\max }^{\text {pow }}\left(s d_{\max }-s d_{\min }\right)+s d_{\text {min }}
$$

This step ensures that the probability of dropping a seed in a distant area decreases nonlinearly with iterations, which results in grouping fitter plants and elimination of inappropriate plants. Therefore, this is a selection mechanism of IWO.

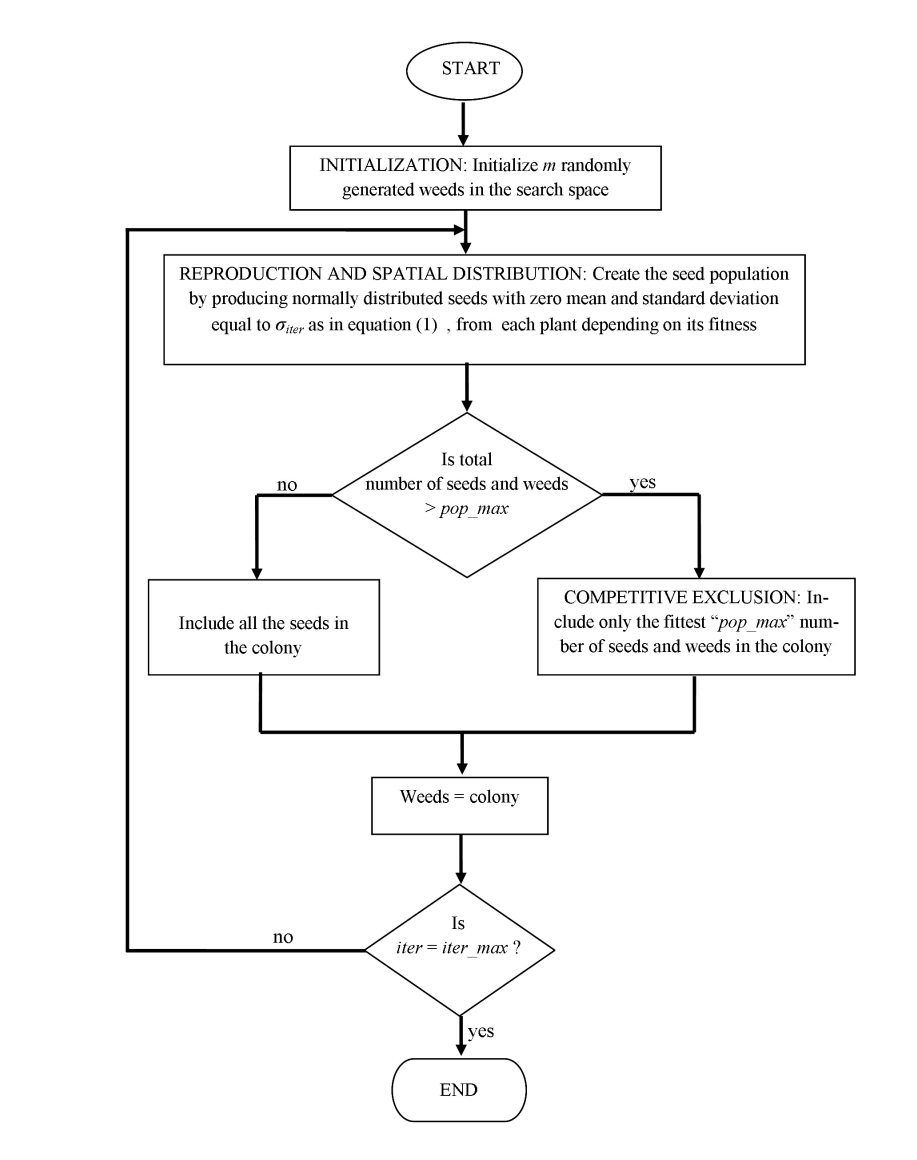

Figure 1: Flow Chart for modified IWO algorithm

\section{Competitive Exclusion:}

If a plant leaves no offspring then it would go extinct, otherwise they would take over the world. Thus, there is a need of some kind of competition between plants for limiting maximum number of plants in a colony. Initially, the plants in a colony will reproduce fast and all the produced plants will be included in the colony, until the number of plants in the colony reaches a maximum value ${ } o p_{\max }$. However, it is expected that by this time the fitter plants have reproduced more than undesirable plants. From then on, only the fittest plants, among the existing ones and the reproduced ones; are taken in the colony and the steps 1 to 4 are repeated until the maximum number of iterations has been reached, i.e. the colony size is fixed from thereon to o $_{\max }$. This method is known as competitive exclusion and is also a selection procedure of IWO. A flowchart of the whole optimization process is illustrated in Figure 1.

Modifications: We have modified (1) as:

$$
s d_{\text {ITER }}=\left(\frac{\text { iter }_{\max }-\text { iter }}{\text { iter }}\right)_{\max }^{\text {pow }} \mid \cos (\text { iter }) \mid\left(s d_{\max }-s d_{\min }\right)+s d_{\min }
$$


The $\mid \cos ($ iter $) \mid$ adds a variation in $s d$ which helps in exploring the better solutions quickly and prevents the new solutions to be spread out of the search space when the $s d$ is relatively large. Suppose we consider an optimization problem where a scalar function $f(\vec{x})$ needs to be minimized. In classical IWO, the seeds are generated from a plant with a certain standard deviation, which is decreased as number of iteration increases. Thus, the plants slowly undergo a behavioral transformation from an explorative nature to an exploitative one. In many engineering problems as this one, often the primary goal is not only to locate the global optima but to find the best possible result utilizing fewer resources. Keeping this in mind the routine of decreasing $s d$ is modified, such that if the weeds are near a suspected optimal solution then it can exploit it quickly rather than wait for the standard deviation to decrease to a reasonable value, which might occur near the end of the run. In our proposed strategy the standard deviation actually varies within an envelope, so lesser values of $s d$ are obtained much before the end of the run. This facilitates quicker detection of optimal solutions and better results as compared to classical IWO, as verified experimentally. Figure 2 illustrates the decrement of $s d$ with iterations for classical IWO and the modified IWO

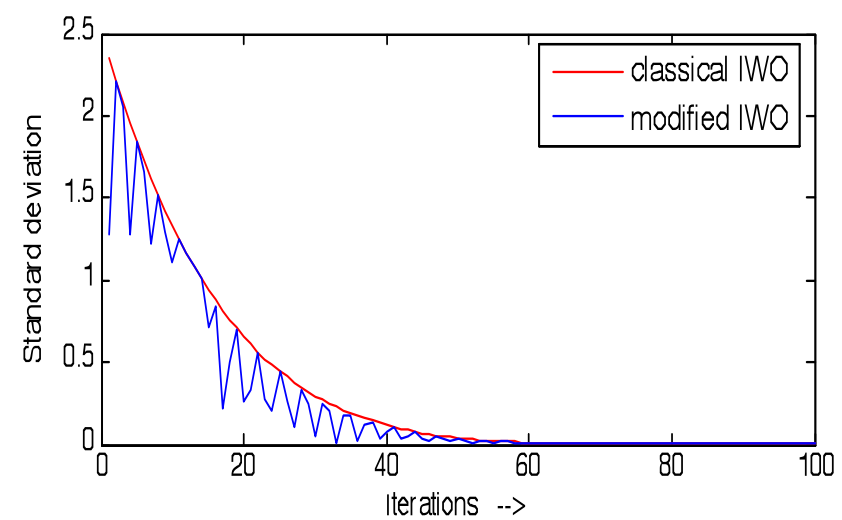

Figure 2: Comparison of the variations of standard deviation with iterations for the classical and modified IWO.

\section{Formulation of Design Problem}

We consider a time modulated linear array of $N$ isotropic elements which are equally spaced and each element is controlled by a high speed radio frequency (RF) switch and excited by complex amplitude. The array is used to transmit a rectangular pulse of width $T$, with a pulse repetition frequency $p r f=1 / T_{p}$, and $T_{p}$ is the pulse repetition period. Here the array factor is given by

$$
F(\theta, t)=e^{j 2 \pi f_{0} t} \cdot \sum_{k=0}^{N} A_{k} e^{j \alpha_{k}} U_{k}(t) e^{j \beta\left(k-\frac{1}{2}\right) d \sin \theta}
$$

where $f_{0}$ and $\beta$ are the centre operating frequency and the wave number in free space, respectively; $\theta$ is the angle measured from the broadside direction; and $d$ is the element spacing. $A_{k}$ and $\alpha_{k}$ are the static excitation amplitude and phase of the $k$ th element, respectively and $U_{k}$ are the periodic "switch-on" time sequence functions in which each element is switched on for $\tau_{k}$ $\left(0 \leq \tau_{k} \leq T\right)$ in each period $T_{p}$.

By decomposing (3) into a Fourier series, the radiation patterns at each harmonic frequency $m . \operatorname{prf}(m=0, \pm 1, \pm \ldots \ldots . \pm \alpha)$ are readily obtained and are given by

$$
F_{n}(\theta, t)=e^{j 2 \pi\left(f_{0}+n f_{p}\right) t} \cdot \sum_{k=1}^{N} a_{n k} e^{j\left[(k-1) \beta d \sin \theta+\alpha_{k}\right]}
$$

where $a_{n k}$ is the complex amplitude and is given by

$$
a_{n k}=A_{k} \tau_{k} f_{p} \cdot \frac{\sin \left[\pi n \tau_{k} f_{p}\right]}{\pi n \tau_{k} f_{p}} \cdot e^{-j \pi n \tau_{k} f_{p}}
$$

At the center frequency $(n=0),(3)$ becomes

$$
a_{0 k}=A_{k} \tau_{k} f_{p}
$$

Thus we can use (5) and (6) to synthesize specific radiation patterns at $f_{0}$ and $f_{0}+p r f$, including ultra-low side lobe levels. To minimize the sideband levels we have used a modified version of the Invasive Weed Optimization (IWO) as the global optimization method. The static excitation amplitudes and the "switch-on" time intervals are the optimization parameter. The cost function is chosen as

$$
\begin{array}{r}
f^{(n)}(v)=\left[w_{1} \cdot \frac{\theta_{B W F N}^{(n)}(v)}{90^{0}}+w_{2} \cdot S L L_{\max }^{(n)}(v)\right]_{f_{0}}+ \\
\left.w_{3} \cdot S B L_{\max }^{(n)}(v)\right|_{f_{0}+p r f}
\end{array}
$$

\section{EXPERIMENTAL RESULTS}

We have considered three different antenna array designs as three problem instantiations. These are 16 element time modulated antenna array, 32 element time modulated antenna array and 64 element time modulated antenna array; among which 32 elements is the most popular case. We have also solved these three problems with three other evolutionary algorithms as DE, DEGL [14] and CLPSO [15]. DEGL is a modified version of the differential evolution algorithm that uses topological neighbourhood (ring-shaped) of the parameter vectors for creating the difference vectors. CLPSO uses a novel learning strategy whereby all other particles' historical best information is used to update a particle's velocity. This strategy enables the diversity of the swarm to be preserved to discourage premature convergence. The parameters for CLPSO and DEGL were chosen as mentioned in [14] and [15] respectively. The detailed parametric setup for all the algorithms is shown in Table 1.

A 16-element linear array of isotropic radiating elements, with $\lambda / 2$ spacing, is considered for the time modulated antenna array with the following parameters: $\mathrm{T}=$ $1 \mu s, p r f=1 \mathrm{MHz}, f_{0}=3.0 \mathrm{GHz}$. Applying Taylor Distribution for determining the excitation amplitudes, the maximum sideband level is about $-17 \mathrm{~dB}$. 
Then the DE algorithm is applied to suppress the sideband levels with the search ranges for the static excitation amplitudes and the "switch on" time intervals are chosen as $[0.252,1.0]$ and $[0.06 \mu \mathrm{s}, 1.0 \mu \mathrm{s}]$ respectively. The maximum side lobe levels found is about $-33.4 \mathrm{~dB}$, which is no doubt an improvement over the Taylor Distribution method. The modified IWO algorithm gives much better results as below:

$\theta_{b w f n}=15.13$ degrees, $\mathrm{SLL}_{\max }=-34.48 \mathrm{~dB}, \mathrm{SBL}_{\max }$ $=-49.67 \mathrm{~dB}$. Moreover to compare the performance of our algorithm we have applied DEGL and CLPSO algorithms to these problems. The power plot for 16 element antenna array is shown in Figures 3, 4, and 5.

\section{Table 1： Parametric Table For Different Algorithms}

( $r_{d}$ is the difference between the maximum and minimum values of the $d$-th decision variable)

\begin{tabular}{|c|c|c|c|c|c|c|c|}
\hline \multicolumn{2}{|c|}{ Modified IWO } & \multicolumn{2}{c|}{ DE } & \multicolumn{2}{c|}{ CLPSO } & \multicolumn{2}{c|}{ DEGL } \\
\hline Param. & Val. & Param & Val & Param. & Val. & Param. & Val. \\
\hline $\begin{array}{c}\text { Maximum } \\
\text { population } \\
\text { size }\end{array}$ & 150 & Pop_size & 150 & $\begin{array}{c}\text { Swarm } \\
\text { size }\end{array}$ & 150 & Pop_size & 150 \\
\hline $\begin{array}{c}\text { Initial } \\
\text { Population } \\
\text { size }\end{array}$ & 50 & $\begin{array}{c}\text { Crossover } \\
\text { Probability } \\
C R\end{array}$ & 0.9 & $C_{1}$ & 1.494 & $\begin{array}{c}\text { Crossove } \\
\text { r } \\
\text { Probabili } \\
\text { ty } C R\end{array}$ & 0.9 \\
\hline $\begin{array}{c}\text { Maximum } \\
\text { number of } \\
\text { seeds }\end{array}$ & 5 & $F$ & 0.8 & $C_{1}$ & 1.494 & $F$ & 0.8 \\
\hline $\begin{array}{c}\text { Minimum } \\
\text { number of } \\
\text { seeds }\end{array}$ & 1 & & Inertial & $\begin{array}{c}\text { linearly } \\
\text { decreased } \\
\text { from } 0.9 \\
\text { to } 0.2\end{array}$ & $\begin{array}{c}\text { Neighbor } \\
\text { hood size }\end{array}$ & $\begin{array}{c}15 \% \text { of } \\
\text { Pop_- } \\
\text { size }\end{array}$ \\
\hline$s d_{\text {max }}$ & $10 \%$ of the search & & $v_{d, \max }$ & $0.9 * r_{d}$ & $\begin{array}{c}\text { weight } \\
\text { factor }\end{array}$ & $\begin{array}{c}\text { fixed, } \\
0.5\end{array}$ \\
\hline$s d_{\text {min }}$ & \multicolumn{2}{|c|}{$\begin{array}{c}0.004 \% \text { of the } \\
\text { search range }\end{array}$} & & & & & \\
\hline$p o w$ & 3 & & & & & & \\
\hline
\end{tabular}

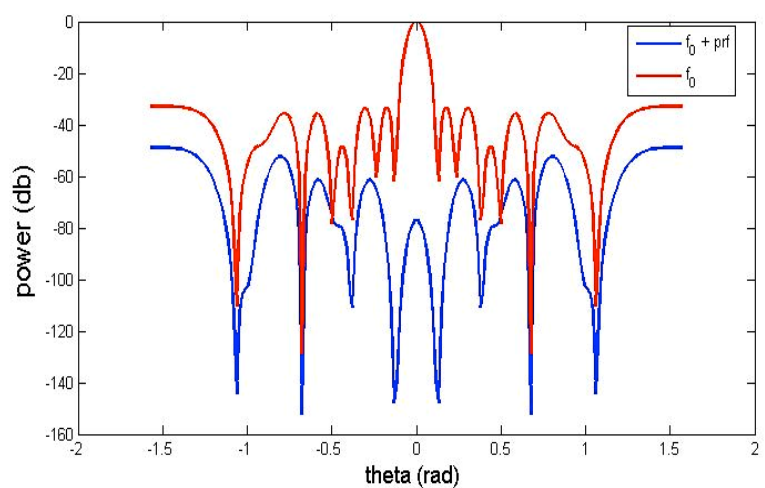

Figure 3: Normalized power patterns of the time modulated linear array with optimized static excitations and switch-on time intervals: $f_{0}$ and $f_{0}+p r f$ by $\mathrm{DE}$ algorithm for 16 element array.

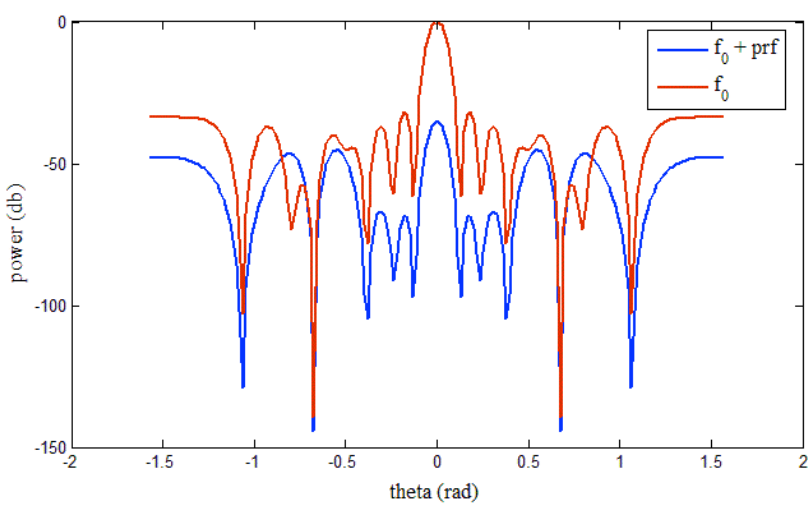

Figure 4: Normalized power patterns of the time modulated linear array with optimized static excitations and switch-on time intervals: $f_{0}$ and $f_{0}+p r f$ by DEGL algorithm for 16 element array.

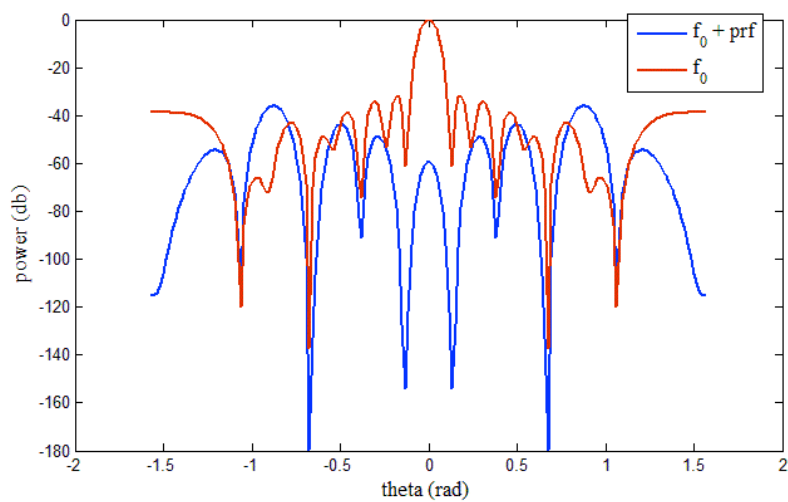

Figure 5: Normalized power patterns of the time modulated linear array with optimized static excitations and switch-on time intervals: $f_{0}$ and $f_{0}+p r f$ by CLPSO algorithm for 16 element array.

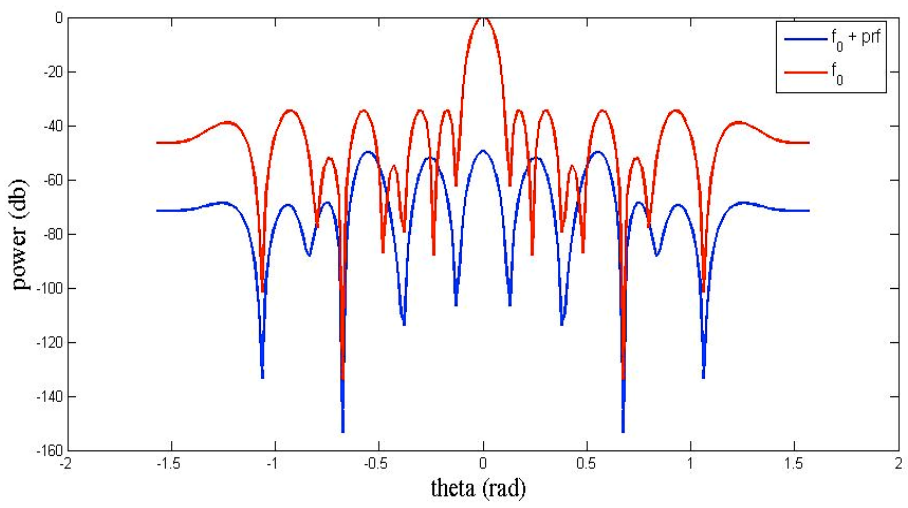

Figure 6: Normalized power patterns of the time modulated linear array with optimized static excitations and switch-on time intervals: $f_{0}$ and $f_{0}+p r f$ by our modified IWO algorithm for 16 element array.

Next the proposed algorithm is applied on a 32 element time modulated linear array with an equal spacing of $\lambda / 2$ operated at $35 \mathrm{GHz}$. The modulation frequency is $1 \mathrm{MHz}$. In these cases 
also the modified IWO algorithm proves better than DE and other algorithms.

The best result found by the three other algorithms DE, CLPSO, DEGL is $\mathrm{SLL}_{\max }=-34.59 \mathrm{~dB}, \mathrm{SBL}_{\max }=-41.83 \mathrm{~dB}$. But we have found much lower $\mathrm{SLL}_{\max }$ as $-36.04 \mathrm{~dB}$ and $\mathrm{SBL}_{\max }=-$ $48.32 \mathrm{~dB}$.

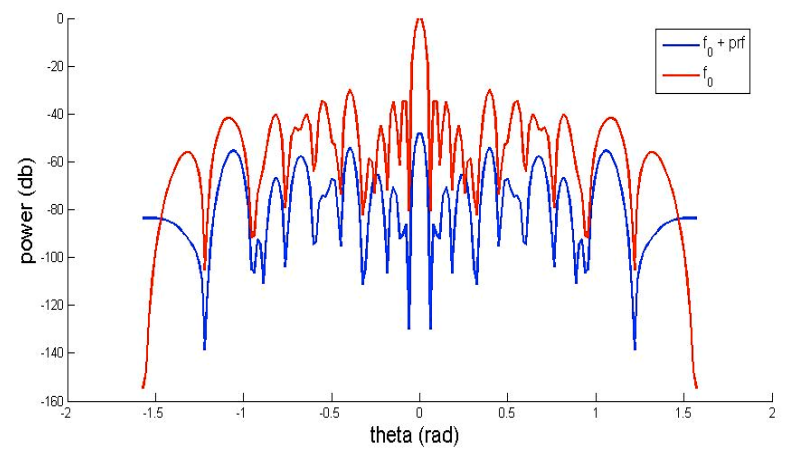

Figure 7: Normalized power patterns of the time modulated linear array with optimized static excitations and switch-on time intervals: $f_{0}$ and $f_{0}+p r f$ by $\mathrm{DE}$ algorithm for 32 element array

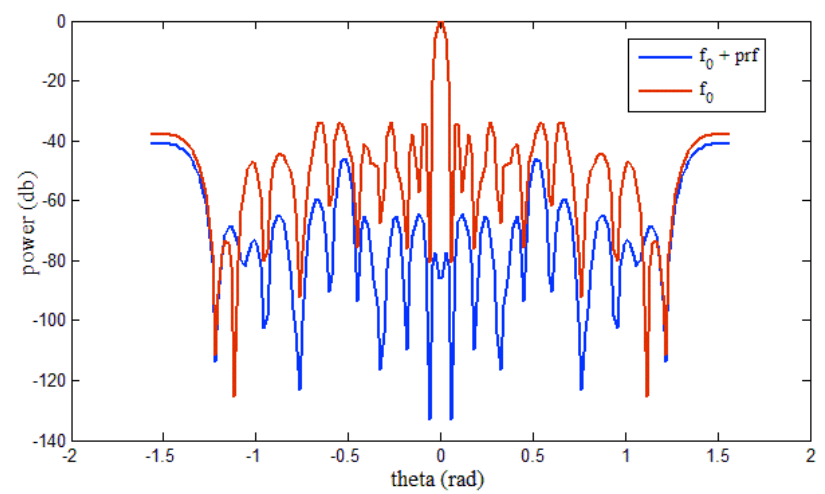

Figure 8: Normalized power patterns of the time modulated linear array with optimized static excitations and switch-on time intervals: $f_{0}$ and $f_{0}+p r f$ by DEGL algorithm for 32 element array

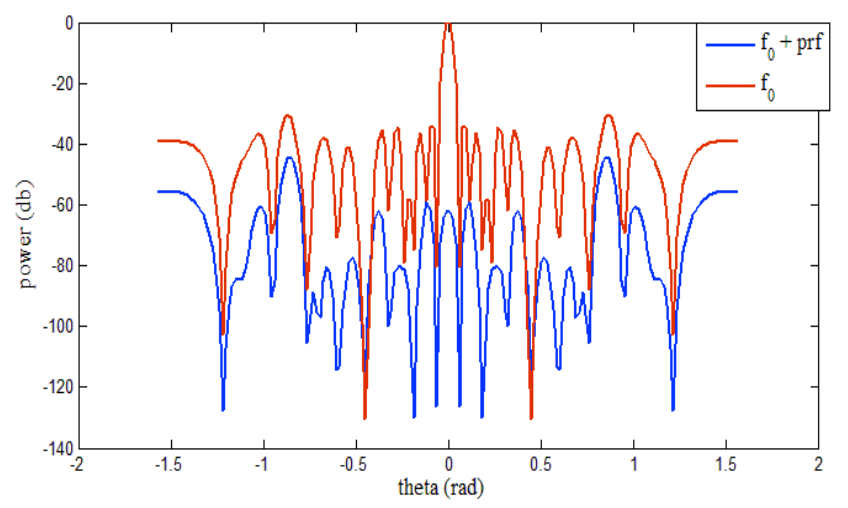

Fig.ure 9: Normalized power patterns of the time modulated linear array with optimized static excitations and switch-on time intervals: $f_{0}$ and $f_{0}+p r f$ by CLPSO algorithm for 32 element array.

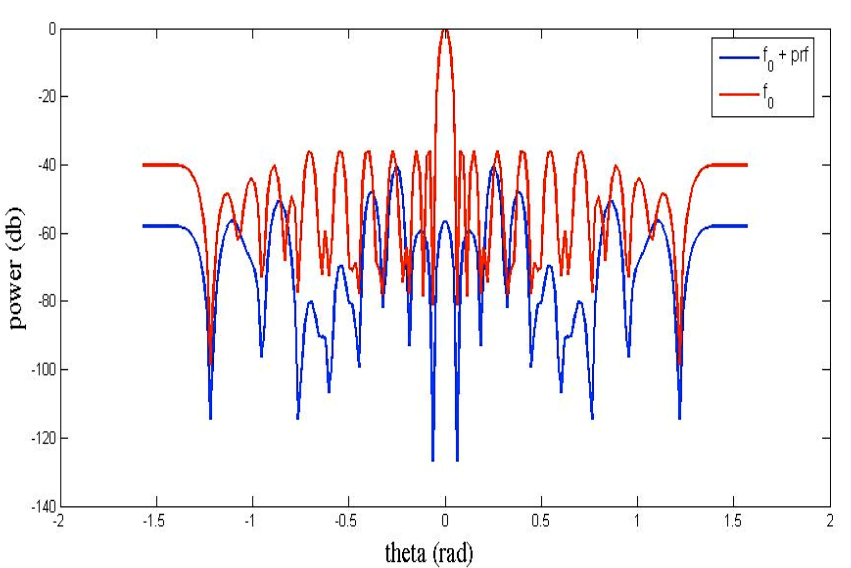

Figure 10: Normalized power patterns of the time modulated linear array with optimized static excitations and switch-on time intervals: $f_{0}$ and $f_{0}+p r f$ by our modified IWO algorithm for 32 element array.

We have also taken the 64 element time modulated antenna array under consideration and suppressed the side lobe levels using our evolutionary algorithm . In this case also the modified IWO algorithm has suppressed the SLLmax and $\theta_{b w f n}$ much compared to other algorithms at $-35.69 \mathrm{~dB}$ and 13.07 degree. Whereas these values are $33.31 \mathrm{~dB}$ and 14.42 degree as found by DEGL, best among the others. Comparison results are shown in the table. The power plot for 64 element antenna array is shown below:

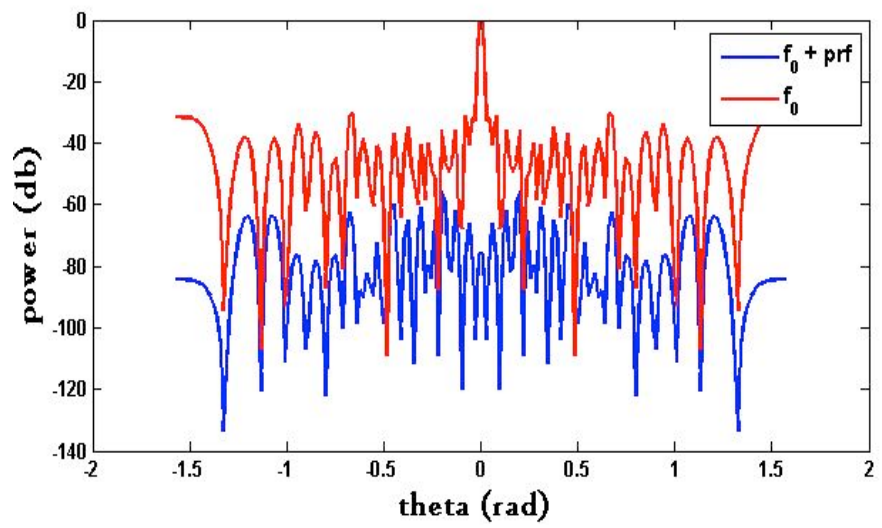

Figure 11: Normalized power patterns of the time modulated linear array with optimized static excitations and switch-on time intervals: $f_{0}$ and $f_{0}+p r f$ by $\mathrm{DE}$ algorithm for 64 element array.

A non-parametric statistical test called Wilcoxon's rank sum test for independent samples $[16,17]$ is conducted at the $5 \%$ significance level in order to judge whether the results obtained with the best performing algorithm differ from the results of the other algorithms in a statistically significant way. $P$ values obtained through the rank sum test between the best algorithm and each of the other algorithms over the three design instances are presented in Table 3. In this table, NA stands for Not Applicable and occurs for the best performing algorithm itself in each case. Also the entries corresponding to statistically insignificant results ( $P$-values greater than 0.05$)$ are marked in bold. If the $P$-values are less than 0.05 (5\% significance level), 
it is a strong evidence against the null hypothesis, indicating that the better final cost function values achieved by the best algorithm in each case is statistically significant and has not occurred by chance.

Table 2 reports the best values of $\mathrm{SLL}_{\max }, \mathrm{SBL}_{\max }$ and $\theta_{b w f n}$ found after fifty independent runs over all the three cases for all algorithms compared. It is clear from the table that suppression of the side lobe levels, which was the main goal of our design, is maximum achieved by our modified IWO algorithm. From the results shown in Table 3 we see that the standard deviation of the cost function value of fifty runs is least in case of our M-IWO algorithm. This indicates the vigorous performance of our algorithm i.e. it produces almost similar results when it is repeatedly applied on the same problem

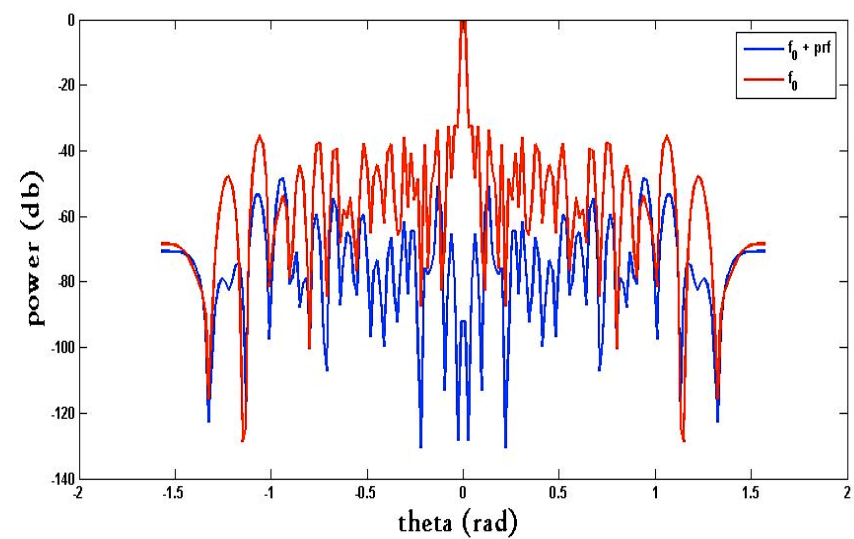

Figure 12: Normalized power patterns of the time modulated linear array with optimized static excitations and switch-on time intervals: $f_{0}$ and $f_{0}+p r f$ by DEGL algorithm for 64 element array.

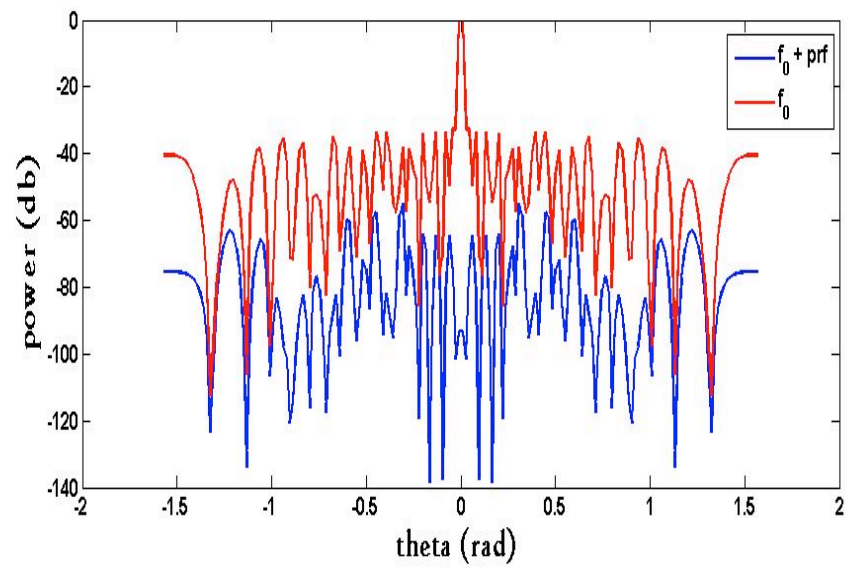

Figure 13: Normalized power patterns of the time modulated linear array with optimized static excitations and switch-on time intervals: $f_{0}$ and $f_{0}+p r f$ by CLPSO algorithm for 64 element array.

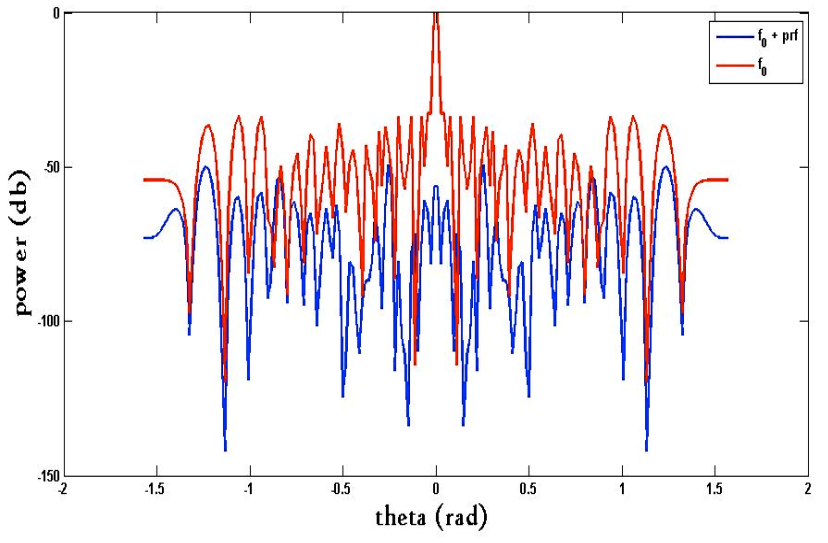

Figure 14: Normalized power patterns of the time modulated linear array with optimized static excitations and switch-on time intervals: $f_{0}$ and $f_{0}+p r f$ by our modified IWO algorithm for 64 element array.

Now to have a comparative measure of the computational time taken by the algorithms we have shown the convergence plots of all the algorithms for the three problem instantiations. As different algorithms evaluate the function value different times in the iterations, variation of fitness value with iteration is not a measure of the computational time consumed by the algorithm. So we have shown the variation of best fitness value with number of function evaluations in Figures 15 ( $a, b$ and $c)$. Here we observe in all the cases, the proposed algorithm has reached the minimum fitness value consuming the least number of FEs, which indicate the greatest convergence speed of the proposed algorithm as compared to the other ones over this design problem. 
TABLE 2: BEST RESULTS OBTAINED BY DIFFERENT ALGORITHMS FOR THREE PROBLEM INSTANCES.

\begin{tabular}{|c|c|c|c|c|c|}
\hline Problem & Algorithm & SLL_max & SBL_max & $\theta_{B W F N}$ & $\begin{array}{c}\text { Weighted Sum }= \\
w 1 * \text { SLL_max }+ \\
w 2 * \text { SBL_max }+ \\
w 3 * \theta_{\text {BWFN }}\end{array}$ \\
\hline \multirow{4}{*}{$\begin{array}{c}16 \text { element } \\
\text { array }\end{array}$} & $\mathrm{DE}$ & -33.4 & -46.2 & 15.06 & -64.54 \\
\hline & DEGL & -31.78 & -31.55 & 15.08 & -48.25 \\
\hline & CLPSO & -31.75 & -32.40 & 15.08 & -49.07 \\
\hline & M-IWO & -34.48 & -49.67 & 15.13 & -69.02 \\
\hline \multirow{4}{*}{$\begin{array}{l}32 \text { element } \\
\text { array }\end{array}$} & $\mathrm{DE}$ & -30.03 & -45.2 & 14.49 & -60.74 \\
\hline & DEGL & -33.94 & -41.83 & 13.06 & -62.70 \\
\hline & CLPSO & -34.59 & -35.93 & 14.44 & -56.08 \\
\hline & M-IWO & -36.04 & -48.32 & 14.42 & -69.94 \\
\hline \multirow{4}{*}{$\begin{array}{l}64 \text { element } \\
\text { array }\end{array}$} & $\mathrm{DE}$ & -28.10 & -50.45 & 14.5 & -64.05 \\
\hline & DEGL & -32.83 & -52.42 & 13.07 & -72.18 \\
\hline & CLPSO & -33.31 & -53.14 & 14.42 & -72.03 \\
\hline & M-IWO & -35.69 & -49.17 & 13.07 & $\begin{array}{l}-71.79 \\
\end{array}$ \\
\hline
\end{tabular}

TABle 3: P-VALUES OBTAINED WITH WILCOXON'S RANK SUM TEST COMPARING THE BEST-PERFORMING ALGORITHM WITH ALL OTHER CONTESTANTS ON THREE DESIGN INSTANCES.

\begin{tabular}{|c|c|c|}
\hline $\begin{array}{c}\text { Number of } \\
\text { Elements }\end{array}$ & Algorithm & $P$-Value \\
\hline \multirow{3}{*}{16} & Modified IWO/DE & $\mathbf{5 . 4 1 2 2 e - 0 1 0}$ \\
\cline { 2 - 3 } & Modified IWO/DEGL & $\mathbf{3 . 5 6 5 4 e - 0 0 6}$ \\
\cline { 2 - 3 } & Modified IWO/CLPSO & $\mathbf{2 . 5 4 7 3 e - 0 0 7}$ \\
\hline \multirow{3}{*}{32} & Modified IWO/DE & $\mathbf{5 . 4 5 7 2 e - 0 1 2}$ \\
\cline { 2 - 3 } & Modified IWO/DEGL & $\mathbf{1 . 7 6 1 2 e - 0 0 5}$ \\
\cline { 2 - 3 } & Modified IWO/CLPSO & $\mathbf{2 . 2 9 1 7 e - 0 0 8}$ \\
\hline \multirow{3}{*}{64} & Modified IWO/DE & $\mathbf{3 . 2 4 2 3 e - 0 0 4}$ \\
\cline { 2 - 3 } & Modified IWO/DEGL & $\mathbf{6 . 4 8 2 1 e - 0 0 3}$ \\
\cline { 2 - 3 } & Modified IWO/CLPSO & $\mathbf{2 . 3 9 4 6 e - 0 0 6}$ \\
\hline
\end{tabular}

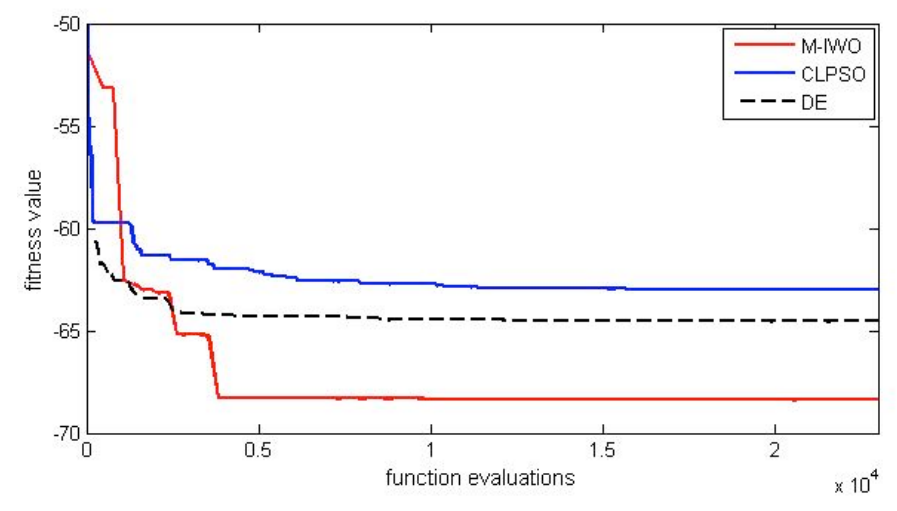

Figure 15 a) 16 element antenna array. 


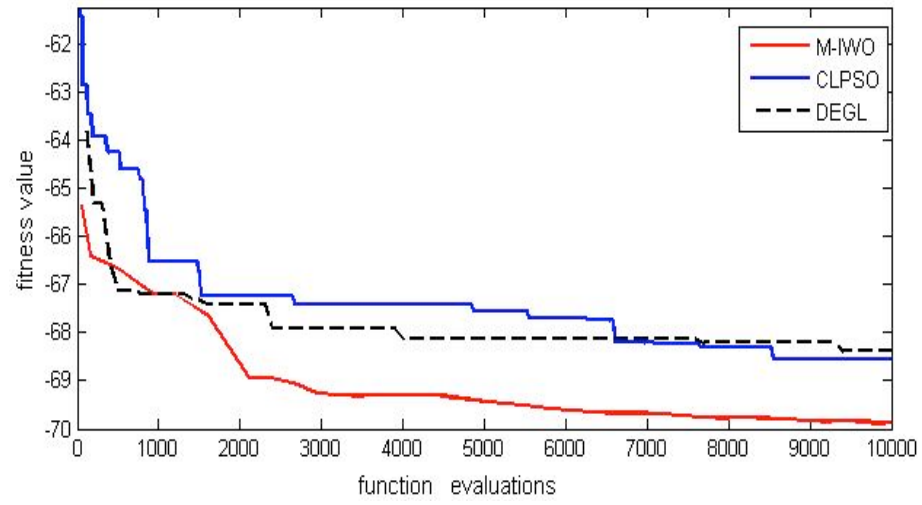

b) 32 element antenna array.

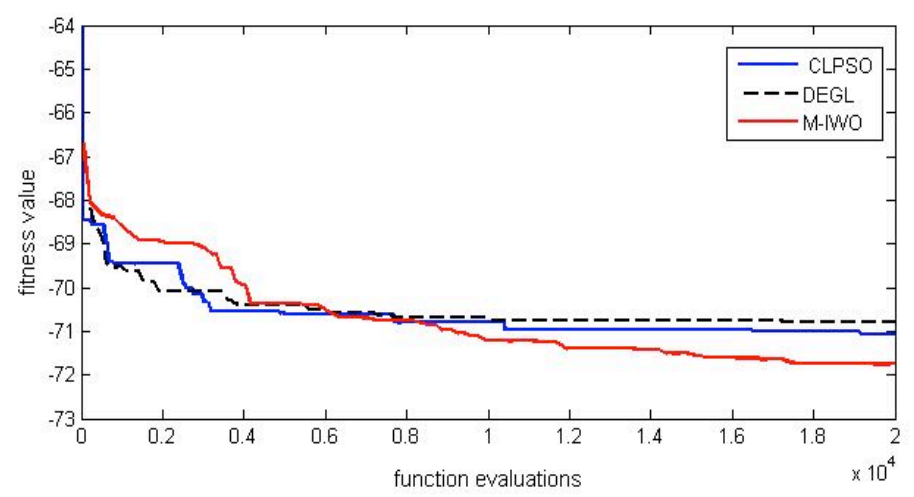

c) 64 element antenna array.

Figure 15. Convergence characteristics of the algorithms

\section{V.CONCLUSIONS}

In this article, we have proposed an improved variant of a recently developed ecologically inspired algorithm called the Invasive Weed Optimization, for the synthesis of timemodulated linear antenna arrays that provide an attractive means for synthesis of low/ultra-low sidelobes. The design problem has been recast as an optimization task, which amounts to minimizing the side lobe levels and side band levels of the antenna array. As evident from the simulation results, the proposed algorithm is statistically significantly better than DE/rand/1/bin, DEGL and CLPSO over the tested design instances. As the M-IWO algorithm has proved much better than the others in terms of both final accuracy and convergence we expect that it will be an attractive alternative tool for different type of antenna synthesis problems such as circular antenna design, monopulse antenna design, X-Band antenna design and so on.

\section{ACKNOWLEDGEMENT}

This work was supported by the Czech Science Foundation under the Grant no.102/09/1494.

\section{REFERENCES}

1. L. C. Godara, Ed., Handbook of Antennas in Wireless Communications, CRC, Boca Raton, FL, 2002.

2. W. H. Kummer, A. T. Villeneuve, T. S. Fong, and F. G. Terrio, "Ultra-low sidelobes from time-modulated arrays," IEEE Trans. Antennas Propag., vol. AP-11, no. 6, pp. 633639, Nov. 1963.

3. H. E. Schrank, "Low sidelobe phased array antennas," IEEE Antennas Propagat. Soc. Newslett., 25 (2), pp. 4-9, 1983.

4. S. Yang, Y. B. Gan, and A. Qing, "Sideband Suppression in Time-Modulated Linear Arrays by the Differential Evolution Algorithm", IEEE Antennas and Wireless Propagation Letters, vol. 1, 2002.

5. R. W. Bickmore, "Time versus space in antenna theory," in Microwave Scanning Antennas, R. C. Hansen, Ed. New York: Academic, vol. III, 1966.

6. Y. Rahmat-Samii and E. Michielssen Eds., Electromagnetic Optimization by Genetic Algorithms. Wiley, 1999.

7. A. R. Mehrabian and C. Lucas, "A novel numerical optimization algorithm inspired from weed colonization," Ecological Informatics, vol. 1, pp. 355-366, 2006.

8. A. R. Mehrabian, A. Yousefi-Koma, "Optimal positioning of piezoelectric actuators on a smart fin using bio-inspired algorithms", Aerospace Science and Technology, vol. 11, pp.174-182, 2007.

9. H. Sepehri Rad, C. Lucas, “A recommender system based on invasive weed optimization algorithm", IEEE Congress on Evolutionary Computation, CEC 2007, pp. 4297-4304.

10. A. R. Mallahzadeh, H. Oraizi, and Z. Davoodi-Rad, "Application of the Invasive Weed Optimization Technique For Antenna Configurations", Progress In Electromagnetics Research PIER 79, 137-150, 2008.

11. A. R. Mallahzadeh, S. Es'haghi, and A. Alipour, "Design of an E-Shaped Mimo Antenna Using IWO Algorithm for Wireless Application at 5.8 GHz", Progress In Electromagnetics Research, PIER 90, 187 - 203, 2009.

12. A. R. Mallahzadeh, S. Es'haghi, and H. R. Hassani, Compact U-array MIMO antenna designs using IWO algorithm, International Journal of RF and Microwave Computer-Aided Engineering, Wiley-InterSscience, DOI: 10.1002/mmce.20379, 2009.

13. X. Zhang, Y. Wang, G. Cui, Y. Niu, and J. Xu, "Application of a novel IWO to the design of encoding sequences for DNA computing", Comput. Math. Appl. 57, pp. 2001-2008, 2009.

14. S. Das, A. Abraham, U. K. Chakraborty, and A. Konar, "Differential evolution using a neighborhood based mutation operator", IEEE Transactions on Evolutionary Computation, 13 (3) pp. 526-553, 2009.

15. J. J. Liang, A. K. Qin, P. N. Suganthan and S. Baskar, "Comprehensive Learning Particle Swarm Optimizer for Global Optimization of Multimodal Functions", IEEE T. on Evolutionary Computation, 10 (3) , pp. 281-295, June 2006.

16. F. Wilcoxon, "Individual comparisons by ranking methods", Biometrics, 1, 80-83, 1945.

17. S. García, D. Molina, M. Lozano, and F. Herrera, "A study on the use of non-parametric tests for analyzing the evolutionary algorithms' behavior: a case study on the CEC'2005 special session on real parameter optimization", Journal of Heuristics, 15 (6) 617-644, 2009. 